\title{
Decomposable Differential Operators in a Cosmological Context
}

\author{
R. Maartens and S. D. Nel \\ Department of Applied Mathematics, University of Cape Town, \\ Rondebosch, Cape Town, South Africa
}

\begin{abstract}
The integrability conditions for a certain second order ordinary differential equation in two variables are studied via the concept of decomposability of the associated differential operator. The results are applied to regain in a unified manner the known exact solutions for locally rotationally symmetric, spatially homogeneous cosmological models. In addition, new solutions are obtained.
\end{abstract}

\section{Introduction}

Einstein's equations

$$
R_{a b}-\frac{1}{2} R g_{a b}+\Lambda g_{a b}=T_{a b}
$$

for a spacetime metric tensor $g_{a b}$, where $R_{a b}$ is the Ricci tensor, $R=R_{a}^{a}$, and $\Lambda$ is the cosmological constant, can be solved exactly only in cases of rather high spacetime symmetry, and for relatively simple forms of the energy-momentum tensor $T_{a b}$.

In this paper, we consider exact solutions of (1.1) for spacetimes in which local coordinates $\left(x^{a}\right)=\left(t, x^{\alpha}\right)(a=0, \ldots, 3 ; \alpha=1, \ldots, 3)$ may be chosen so that one or more of the field equations, or combinations thereof, take the generic form

$$
A_{1} \frac{\ddot{X}}{X}+A_{2} \frac{\ddot{Y}}{Y}+A_{3} \frac{\dot{X}^{2}}{X^{2}}+A_{4} \frac{\dot{Y}^{2}}{Y^{2}}+A_{5} \frac{\dot{X}}{X}+A_{6} \frac{\dot{Y}}{Y}+A_{7} \frac{\dot{X} \dot{Y}}{X Y}+H(X, Y, t)=0
$$

where $A_{i} \in \mathbf{R}(i=1, \ldots, 7)$, a dot denotes differentiation with respect to $t$, and $X(t), Y(t)$ are metric component functions. This is the case, for example, when the spacetime is locally rotationally symmetric and admits a 1-parameter family of homogeneous hypersurfaces (see, e.g., Refs. [1-3]). If a first integral of (1.2) can be found, then together with the remaining field equations and the conservation equations $T_{; b}^{a b}=0$, this often allows us to obtain a reduction of the system of equations to quadratures, or to a single ordinary differential equation in one variable, plus quadratures. 
To study the integrability conditions for (1.2), we define a differential operator on functions of differentiability class $C^{k}(k \geqq 2)$

$$
L_{\mathbf{a}}: C^{k}(\mathbf{R}) \times C^{k}(\mathbf{R}) \rightarrow C^{k-2}(\mathbf{R})
$$

by

$$
\begin{aligned}
L_{\mathrm{a}}[u, v](t)= & a_{1} \ddot{u}(t)+a_{2} \ddot{v}(t)+a_{3} \dot{u}^{2}(t)+a_{4} \dot{v}^{2}(t) \\
& +a_{5} \dot{u}(t)+a_{6} \dot{v}(t)+a_{7} \dot{u}(t) \dot{v}(t)
\end{aligned}
$$

where $a_{1}^{2}+a_{2}^{2} \neq 0$.

$L_{\mathrm{a}}$ will be said to be decomposable if there exist a function $F=F(u, v, t)$, of class $C^{k}$ in its arguments, and a function $G=G(u, \dot{u}, v, \dot{v}, t)$, of class $C^{k+1}$ in its arguments, such that

$$
L_{\mathrm{a}}[u, v](t)=F(u, v, t) \dot{G}(u, \dot{u}, v, \dot{v}, t) .
$$

If $L_{\mathrm{a}}$ is decomposable, then the differential equation

$$
L_{\mathrm{a}}[u, v](t)=0
$$

is integrable, with integrating factor $F^{-1}$. In addition, (1.2), which is of form

$$
L_{\mathrm{a}}[u, v](t)+H(u, v, t)=0
$$

where $u=\log |X|, v=\log |Y|$, and $\mathbf{a}=\left(A_{1}, A_{2}, A_{3}+A_{1}, A_{4}+A_{2}, A_{5}, A_{6}, A_{7}\right)$, then has a first integral

$$
G\left(u, \frac{d u}{d t^{\prime}}, v, \frac{d v}{d t^{\prime}}, t\left(t^{\prime}\right)\right)=t_{0}^{\prime}-t^{\prime} \quad\left(t_{0}^{\prime} \in \mathbf{R}\right)
$$

where

$$
t^{\prime}(t)=\int \frac{H(u(t), v(t), t)}{F(u(t), v(t), t)} d t .
$$

In $\S 2$, the complete characterisation of decomposability of operators of form (1.3), is found. Although this does not lead to the full integrability conditions for (1.5) (since in general an integrating factor for (1.5) will depend also on $\dot{u}$ and $\dot{v}$ ), it is sufficient to lead to the regaining of all known exact solutions for perfect fluid spacetimes which are locally rotationally symmetric and spatially homogeneous (as listed by MacCallum [4]). Together with these known solutions, new exact solutions are given in $\S 3$. $\S 4$ contains some remarks on possible extensions of the method.

\section{Integrability Conditions}

Theorem 2.1. $L_{\mathrm{a}}$ is decomposable iff

$$
\begin{aligned}
& a_{1}^{2} a_{4}+a_{2}^{2} a_{3}-a_{1} a_{2} a_{7}=0 \\
& a_{5}^{2} a_{4}+a_{6}^{2} a_{3}-a_{5} a_{6} a_{7}=0 .
\end{aligned}
$$


Proof. Necessity. Suppose (1.4) holds. Then, by (1.3)

$$
\begin{aligned}
& F \frac{\partial G}{\partial \dot{u}}=a_{1} \\
& F \frac{\partial G}{\partial \dot{v}}=a_{2} \\
& F\left[\frac{\partial G}{\partial u} \dot{u}+\frac{\partial G}{\partial v} \dot{v}+\frac{\partial G}{\partial t}\right]=a_{3} \dot{u}^{2}+a_{4} \dot{v}^{2}+a_{5} \dot{u}+a_{6} \dot{v}+a_{7} \dot{u} \dot{v} .
\end{aligned}
$$

By differentiating (2.1) with respect to $\dot{u}$ and $\dot{v}$ we obtain

$$
\begin{aligned}
& F \frac{\partial^{2} G}{\partial \dot{u} \partial u}=a_{3} \\
& F \frac{\partial^{2} G}{\partial \dot{v} \partial v}=a_{4} \\
& F\left[\frac{\partial^{2} G}{\partial \dot{u} \partial v}+\frac{\partial^{2} G}{\partial \dot{v} \partial u}\right]=a_{7} \\
& \frac{\partial}{\partial t}\left[G-\frac{\partial G}{\partial \dot{u}} \dot{u}-\frac{\partial G}{\partial \dot{v}} \dot{v}\right]=0 .
\end{aligned}
$$

Case 1: $a_{1} a_{2} \neq 0$

By differentiating (2.1a), (2.1b) with respect to $u, v$ (respectively), and using (2.2a), (2.2b), we obtain expressions, which, with (2.2c), yield (D1).

Also, these expressions, together with (2.2) and (D1), give expressions for $a_{5}, a_{6}$, which, when differentiated with respect to $v, u$ (respectively), and subtracted, yield

$$
a_{2} a_{3} a_{6}-a_{1} a_{4} a_{5}=\left[a_{1}^{2} a_{4}-a_{2}^{2} a_{3}\right] \frac{\partial}{\partial t} \log F
$$

while differentiation with respect to $t$ gives, using (2.1a), (2.1b) and (2.2d), and subtracting

$$
\left(a_{2} a_{5}-a_{1} a_{6}\right) \frac{\partial F}{\partial t}=0 .
$$

Hence

$$
\left(a_{2} a_{5}-a_{1} a_{6}\right)\left(a_{2} a_{3} a_{6}-a_{1} a_{4} a_{5}\right)=0
$$

(D2) follows from (D1) and (2.3).

Case 2: $a_{2}=0$

By (2.1b) and (2.2b)

$$
a_{4}=0
$$


which is just (D1) for $a_{2}=0$.

(D2) is obtained by steps similar to those in Case 1.

\section{Sufficiency}

Case 1: $a_{1} a_{2} \neq 0$

Case 1(a): $a_{3}=0=a_{4}$

(D1) implies $a_{7}=0$, and hence we can set

$$
\begin{aligned}
& F=1 \\
& G=a_{1} \dot{u}+a_{2} \dot{v}+a_{5} u+a_{6} v .
\end{aligned}
$$

Case 1(b): $a_{3}^{2}+a_{4}^{2} \neq 0$

(D1), (D2) imply (2.3), and hence we can set

$$
\begin{aligned}
& F=\exp \left[\alpha t-\frac{a_{3}}{a_{1}} u-\frac{a_{4}}{a_{2}} v\right] \\
& G=F^{-1}\left(a_{1} \dot{u}+a_{2} \dot{v}+\beta\right)
\end{aligned}
$$

where $\alpha$ and $\beta$ are defined as follows:

$$
\begin{aligned}
& \text { if } a_{2} a_{5}-a_{1} a_{6}=0 \text { then } \alpha=-\frac{a_{5}}{a_{1}}=-\frac{a_{6}}{a_{2}} \text { and } \beta=0 \\
& \text { if } a_{2} a_{5}-a_{1} a_{6} \neq 0 \text { then } \alpha=0 \text { and } \beta=\left\{\begin{array}{l}
\frac{a_{1} a_{5}}{a_{3}} \text { if } a_{3} \neq 0 \\
\frac{a_{2} a_{6}}{a_{4}} \text { if } a_{4} \neq 0 .
\end{array}\right.
\end{aligned}
$$

The remaining case (Case 2: $a_{2}=0$; Case 2(a): $a_{3}=0=a_{7}$, Case 2(b): $a_{3}^{2}+$ $\left.a_{7}^{2} \neq 0\right)$ is treated similarly.

In all cases we obtain $F(u, v, t) \dot{G}(u, \dot{u}, v, \dot{v}, t)=L_{\mathrm{a}}[u, v](t)$.

We now investigate the behaviour of the decomposability conditions (D1), (D2) under transformation of variable. Define differentiable functions

$$
D_{\sigma}: \mathbf{R}^{7} \rightarrow \mathbf{R} \quad(\sigma=1,2)
$$

by

$$
\begin{aligned}
& D_{1}\left(x^{i}\right)=\left(x^{1}\right)^{2} x^{4}+\left(x^{2}\right)^{2} x^{3}-x^{1} x^{2} x^{7} \\
& D_{2}\left(x^{i}\right)=\left(x^{1}\right)^{2} x^{4}+\left(x^{6}\right)^{2} x^{3}-x^{5} x^{6} x^{7}
\end{aligned}
$$

where

$$
\left(x^{1}\right)^{2}+\left(x^{2}\right)^{2} \neq 0 .
$$

Then

$$
\mathscr{D}=D_{1}^{-1}(0) \cap D_{2}^{-1}(0)
$$


is a 6-dimensional differentiable variety of $\mathbf{R}^{7}$, and $\left\{L_{\mathbf{a}} \mid \mathbf{a} \in \mathscr{D}\right\}$ is, by Theorem 2.1, the set of all decomposable operators $L_{\mathbf{a}}$.

A transformation of variable $(u, v, t) \mapsto\left(u^{\prime}, v^{\prime}, t^{\prime}\right)$, given by

$$
\begin{aligned}
& u=\phi^{1}\left(u^{\prime}, v^{\prime}, t^{\prime}\right) \\
& v=\phi^{2}\left(u^{\prime}, v^{\prime}, t^{\prime}\right) \\
& t=\phi^{3}\left(u^{\prime}, v^{\prime}, t^{\prime}\right)
\end{aligned}
$$

results in a transformation

$$
L_{\mathrm{a}}[u, v](t) \mapsto M\left(u^{\prime}, \frac{d u^{\prime}}{d t^{\prime}}, \frac{d^{2} u^{\prime}}{d t^{\prime 2}}, v^{\prime}, \frac{d v^{\prime}}{d t^{\prime}}, \frac{d^{2} v^{\prime}}{d t^{\prime 2}}, t^{\prime}\right) .
$$

If

$$
M\left(u^{\prime}, \ldots, t^{\prime}\right)=L_{\mathbf{a}^{\prime}}\left[u^{\prime}, v^{\prime}\right]\left(t^{\prime}\right)
$$

where $\mathbf{a}^{\prime} \in \mathbf{R}^{7}$, the transformation will be said to be form-preserving. Let $\mathscr{G}$ denote the group of form-preserving transformations, and denote by $\mathscr{G}^{*}$ the group of parameter transformations $\mathbf{a} \mapsto \mathbf{a}^{\prime}$ of $\mathbf{R}^{7}$ induced by $\mathscr{G}$. (2.4b) implies that $\phi^{3}=$ $\phi^{3}\left(t^{\prime}\right)$ and hence it is shown readily that $\mathscr{G}$ preserves the property of decomposability. $\mathscr{D}$ is therefore an invariant variety of $\mathscr{G}^{*}:$ if $L_{\mathrm{a}}$ is not decomposable, no form-preserving transformation (2.4a) of the variables can render it decomposable.

(Explicitly, $\mathscr{G}$ is given locally by the transformation equations

$$
\begin{aligned}
& u=g^{1} u^{\prime}+g^{2} v^{\prime}+g^{6} t^{\prime}+g^{8} \\
& v=g^{3} u^{\prime}+g^{4} v^{\prime}+g^{7} t^{\prime}+g^{9} \\
& t=g^{5} t^{\prime}+g^{10}
\end{aligned}
$$

where $g^{A} \in \mathbf{R}(A=1, \ldots, 10),\left(g^{1} g^{4}-g^{2} g^{3}\right) g^{5} \neq 0$, and

$$
L_{\mathrm{a}}\left[\left(\frac{g^{6}}{g^{5}}\right) t,\left(\frac{g^{7}}{g^{5}}\right) t\right]=0 .
$$

Then

$$
\begin{aligned}
& D_{1}\left(a_{i}^{\prime}\right)=\left(g^{1} g^{4}-g^{2} g^{3}\right)^{2}\left(g^{5}\right)^{5} D_{1}\left(a_{i}\right) \\
& D_{2}\left(a_{i}^{\prime}\right)=\left(g^{1} g^{4}-g^{2} g^{3}\right)^{2}\left(g^{5}\right)^{4} D_{2}\left(a_{i}\right)+\left(4 a_{3} a_{4}-a_{7}^{2}\right) L_{\mathrm{a}}\left[\left(\frac{g^{6}}{g^{5}}\right) t,\left(\frac{g^{7}}{g^{5}}\right) t\right]
\end{aligned}
$$

verifying that $\mathscr{D}$ is an invariant variety of $\mathscr{G}^{*}$ ).

\section{Examples}

In this section we show, by means of illustrative examples, how in a cosmological context the foregoing results sometimes allow a reduction of Einstein's field equations to one or more ordinary differential equations involving only one dependent variable. We shall assume throughout that the large scale matter and 
radiation distribution in the universe is represented by a perfect fluid obeying the equation of state $p=(\gamma-1) \mu$, where $1 \leqq \gamma \leqq 2$.

\section{Example 1: Orthogonal, Locally Rotationally Symmetric,} Spatially Homogeneous Models [1]

For these models, there exist co-moving co-ordinates $(t, x, y, z)$ such that

$$
d s^{2}=-d t^{2}+X^{2}(t) d x^{2}+Y^{2}(t)\left[d y^{2}+f^{2}(y) d z^{2}\right]-X^{2}(t) h(y)[2 d x-h(y) d z] d z
$$

where

$$
f(y)=\left[\begin{array}{c}
\sin y \\
y \\
\sinh y
\end{array}\right], h(y)=\left[\begin{array}{c}
2 c \cos y \\
-c^{2} y^{2} \\
-2 c \cosh y
\end{array}\right] \text { for } k=\left[\begin{array}{r}
1 \\
0 \\
-1
\end{array}\right]
$$

and $c, k \in \mathbf{R}$ are parameters related to the symmetry group of the space-time.

The fluid 4-velocity is $\mathbf{u}=\frac{\partial}{\partial t}$, and the field equations are

$$
\begin{aligned}
& \frac{\ddot{X}}{X}+\frac{\dot{X} \dot{Y}}{X Y}+\frac{\ddot{Y}}{Y}+c^{2} \frac{X^{2}}{Y^{4}}=(1-\gamma) \mu+\Lambda \\
& 2 \frac{\ddot{Y}}{Y}+\frac{\dot{Y}^{2}+k}{Y^{2}}-3 c^{2} \frac{X^{2}}{Y^{4}}=(1-\gamma) \mu+\Lambda \\
& 2 \frac{\dot{X} \dot{Y}}{X Y}+\frac{\dot{Y}^{2}+k}{Y^{2}}-c^{2} \frac{X^{2}}{Y^{4}}=\mu+\Lambda
\end{aligned}
$$

while the conservation equations reduce to

$$
\frac{1}{\gamma} \frac{\dot{\mu}}{\mu}+\frac{\dot{X}}{X}+2 \frac{\dot{Y}}{Y}=0
$$

Using the reduction technique described in this paper, we have been able to regain all the hitherto known exact solutions of (3.1) (of which we are aware [4]). These are:

$c=0$ : Bianchi $I(k=0)$ : General solutions for $1 \leqq \gamma \leqq 2$; Vacuum.

Kantowski-Sachs $\mathrm{I}(k=+1), \operatorname{II}(k=-1)$ : General solutions for $\gamma=1, \frac{4}{3}(\Lambda=$ $0), 2(\Lambda=0)$; Vacuum.

$c \neq 0$ : Bianchi $\mathrm{II}(k=0)$ : Special solutions for $1 \leqq \gamma \leqq 2$; General solution for $\gamma=2(\Lambda=0)$; Vacuum.

Bianchi $\operatorname{VIII}(k=-1), \operatorname{IX}(k=+1)$ : Vacuum.

Detailed references may be found in [4].

In addition, we have obtained the (apparently new) general solution for the case $c k \neq 0, \gamma=2$ (Bianchi types VIII and IX) and a general reduction to one second order o.d.e. and two quadratures for the case $c \neq 0, k=0$ (Bianchi II) (in both cases with $\Lambda=0$ ). These are as follows. 
Case (i): $c k \neq 0, \gamma=2$. (Bianchi types VIII and IX)

(3.1a) and (3.1c) decompose to

$\left[Y(X Y)^{\circ}\right]^{\cdot}=-k X$.

If we define a new time parameter $\tau$ by

$\tau(t)=\int X(t) d t$

(3.2) may be integrated twice to give

$(X Y)^{2}=-k \tau^{2}+a \tau+b$.

Substituting for $\mu=d^{2} X^{-2} Y^{-4}(d \in \mathbf{R})$ from (3.1d) and for $Y$ from (3.4) into (3.1c), yields an equation for $X$ :

$$
\left[\left(X^{-2}\right)^{\prime}\right]^{2}=\left[\left(a^{2}+4 b k-d^{2}\right) X^{-2}-4 c^{2}\right]\left(-k \tau^{2}+a \tau+b\right)^{-2}
$$

where ${ }^{\prime} \equiv \frac{d}{d \tau}$.

Since (3.5) is separable it integrates immediately to give $X=X(\tau)$, and then $Y=Y(\tau)$ is found from (3.4).

Thus the full solution is obtained in parametric form, where the proper time $t$ is related to the parameter $\tau$ by (3.3).

Note that the vacuum solution $(\mu=0)$ is given by setting $d=0$ in (3.5).

Case (ii): $c \neq 0, k=0$. (Bianchi II)

Using (3.1a) and (3.1c) as before, we find after substituting for $\mu$ from (3.1d) and decomposing

$$
\left[Y(X Y)^{\cdot}\right]^{\cdot}=(2-\gamma) d^{2}\left(X Y^{2}\right)^{1-\gamma}
$$

If $\gamma=2$, we proceed as in (i) and obtain (3.5) with $k=0$.

If $1 \leqq \gamma<2$, we define a new time parameter $\eta$ by

$$
\eta=\left[(2-\gamma) d^{2(3-\gamma)}\right]^{1 / 2} \int\left[X(t) Y^{2}(t)\right]^{1-\gamma} d t .
$$

Then (3.6) may be integrated to give

$$
\frac{X^{\prime}}{X}+\frac{Y^{\prime}}{Y}=\mu^{(2-\gamma) / \gamma}\left(\eta-\eta_{0}\right)
$$

where $^{\prime} \equiv \frac{d}{d \eta}$ and $\quad \eta_{0} \in \mathbf{R}$.

Now (3.8) and (3.1d) allow us to express $\frac{X^{\prime}}{X}$ and $\frac{Y^{\prime}}{Y}$ purely in terms of $\mu$. Substituting this into the linear combination (3.1) [(b)-3(c)], we obtain a second order equation for $\mu$ in terms of $\eta$ :

$$
\begin{aligned}
& \mu \mu^{\prime \prime}-(4 / \gamma) \mu^{\prime 2}-8\left(\eta-\eta_{0}\right) \mu^{2 / \gamma} \mu^{\prime}-6 \gamma\left(\eta-\eta_{0}\right)^{2} \mu^{4 / \gamma}+[\gamma-\gamma(\gamma+2) / 2(2-\gamma) \\
& \left.\cdot d^{2(3-\gamma)}\right] \mu^{(2+\gamma) / \gamma}=0 .
\end{aligned}
$$

After solving (3.9) to obtain $\mu=\mu(\eta)$, the solution is completed by the quadratures 


$$
\begin{aligned}
& X=a \mu^{1 / \gamma} \exp \left[2 \int \mu^{(2-\gamma) / \gamma}\left(\eta-\eta_{0}\right) d \eta\right] \\
& Y=b \mu^{-1 / \gamma} \exp \left[-\int \mu^{(2-\gamma) / \gamma}\left(\eta-\eta_{0}\right) d \eta\right]
\end{aligned}
$$

where $a, b \in \mathbf{R}$.

\section{Example 2: Tilted, Locally Rotationally Symmetric,} Spatially Homogeneous Models [2]

For these models, (invariant under a group of Bianchi type V), there exist coordinates $(t, x, y, z)$ such that

$$
d s^{2}=-d t^{2}+X^{2}(t) d x^{2}+Y^{2}(t) \exp \left(-2 A_{0} x\right)\left(d y^{2}+d z^{2}\right)
$$

where $A_{0} \in \mathbf{R}, A_{0} \neq 0$, and the fluid 4-velocity is

$$
\mathbf{u}=(\cosh \psi) \frac{\partial}{\partial t}+\left(X^{-1} \sinh \psi\right) \frac{\partial}{\partial x},
$$

where $\psi$ is the hyperbolic tilt angle.

The field equations are (with $\Lambda=0$ )

$$
\begin{aligned}
& \frac{\ddot{X}}{X}+2 \frac{\dot{X} \dot{Y}}{X Y}-2 \frac{A_{0}^{2}}{X^{2}}=\frac{1}{2}(2-\gamma) \mu+\gamma \mu \sinh ^{2} \psi \\
& \frac{\ddot{Y}}{Y}+\frac{\dot{Y}^{2}}{Y^{2}}+\frac{\dot{X} \dot{Y}}{X Y}-2 \frac{A_{0}^{2}}{X^{2}}=\frac{1}{2}(2-\gamma) \mu \\
& \frac{2 A_{0}}{X}\left(\frac{\dot{X}}{X}-\frac{\dot{Y}}{Y}\right)=\gamma \mu \sinh \psi \cosh \psi \\
& \frac{\dot{Y}^{2}}{Y^{2}}+2 \frac{\dot{X} \dot{Y}}{X Y}-3 \frac{A_{0}^{2}}{X^{2}}=\mu \cosh ^{2} \psi+(\gamma-1) \mu \sinh ^{2} \psi
\end{aligned}
$$

while the conservation equations can be written as

$$
\left[\log \left(\mu^{\gamma} X Y^{2} \cosh \psi\right)\right]^{\cdot}=\frac{2 A_{0}}{X} \tanh \psi
$$

$\left[\log \left(\mu^{(\gamma-1) / \gamma} X \sinh \psi\right)\right]^{*}=0$.

We shall solve these equations for the case $\gamma=2$. This appears to be the first exact solution for this class of model with non-vanishing pressure.

By (3.10e),

$$
\mu=A_{0}^{2}(\alpha \sinh \psi X)^{-2}
$$

where $\alpha \in \mathbf{R}$.

Substituting (3.11) into (3.10a) and (3.10b), we find

$$
\alpha^{2} \frac{\ddot{X}}{X}-\left(\alpha^{2}+1\right) \frac{\ddot{Y}}{Y}-\left(\alpha^{2}+1\right) \frac{\dot{Y}^{2}}{Y^{2}}+\left(\alpha^{2}-1\right) \frac{\dot{X} \dot{Y}}{X Y}=0 .
$$


By rescaling the x-coordinate, we may set $\alpha^{2}=1$, in which case (3.12) satisfies the conditions of Theorem 2.1, and decomposes to

$$
\left[X^{2}\left(X^{-1} Y^{2}\right)^{\cdot}\right]^{\cdot}=0 \text {. }
$$

Furthermore, (3.10b) decomposes to

$$
\left[X\left(Y^{2}\right)^{\circ}\right]^{\cdot}=2 A_{0}^{2} Y^{2} X^{-1}
$$

which, after the introduction of a new time parameter $\xi$, defined by

$$
\xi(t)=\int X^{-1}(t) d t
$$

becomes

$$
\left(Y^{2}\right)^{\prime \prime}-2 A_{0}^{2} Y^{2}=0
$$

where ${ }^{\prime} \equiv \frac{d}{d \xi}$.

Hence

$$
Y^{2}=a \sinh \left(2 A_{0} \xi\right)+b \cosh \left(2 A_{0} \xi\right)
$$

$a, b \in \mathbf{R}$, and by (3.13)

$$
X=c Y^{2} \exp \left(-d \int Y^{-2}(\xi) d \xi\right)
$$

where $c, d \in \mathbf{R}$.

The hyperbolic tilt angle $\psi$ can be found from (3.10c), (3.14) and (3.16) to be

$$
\psi=\operatorname{arc} \operatorname{coth}\left[Y^{-2}\left(Y Y^{\prime}+d\right)\right]
$$

and now $\mu$ is determined by (3.11).

As in Example 1, the full solution is thereby obtained in parametric form.

The Farnsworth dust solution $(\gamma=1)$ [5] can be regained by our method after changing to comoving coordinates $(\tau, x, y, z)$ in which

$$
d s^{2}=-d \tau^{2}+2 F(\tau) d \tau d x+\left[W^{2}(\tau)-F^{2}(\tau)\right] d x^{2}+Y^{2}(\tau) \exp \left(-2 A_{0} x\right)\left[d y^{2}+d z^{2}\right]
$$

and $\mathbf{u}=\frac{\partial}{\partial \tau}$.

Example 3: Tilted, Non-Locally Rotationally Symmetric, Spatially Homogeneous Models [2]

The method we have developed can be extended easily to the case where the generic Equation (1.2) contains more than 2 dependent variables. Even without this extension, however, it is sometimes possible to obtain the necessary decomposition by defining new variables in such a way that the problem involves essentially only 2 dependent variables.

For example, a certain class (the case Bianchi II, $\Sigma_{23}=0$, in the notation of [2]) of tilted, spatially homogeneous models may be given by

$$
d s^{2}=-d t^{2}+X^{2}(t)[d x-f(t, z) d y]^{2}+Y^{2}(t) d y^{2}+Z^{2}(t) d z^{2}
$$


where

$$
f(t, z)=-\sqrt{2 a} Z-\sqrt{2 b} \int Y(t)\left[X^{3}(t) Z(t)\right]^{-1} d t
$$

$a, b>0$, and the fluid 4-velocity is

$$
\mathbf{u}=\cosh \psi \frac{\partial}{\partial t}+Z^{-1} \sinh \psi \frac{\partial}{\partial z} .
$$

The field equations are (with $\Lambda=0$ )

$$
\begin{aligned}
& \frac{\ddot{X}}{X}+\frac{\dot{X} \dot{Y}}{X Y}+\frac{\dot{X} \dot{Z}}{X Z}+a\left(\frac{X}{Y Z}\right)^{2}-\frac{b}{X^{4} Z^{2}}=\frac{1}{2}(2-\gamma) \mu \\
& \frac{\ddot{Y}}{Y}+\frac{\dot{Y} \dot{Z}}{Y Z}+\frac{\dot{X} \dot{Y}}{X Y}-a\left(\frac{X}{Y Z}\right)^{2}+\frac{b}{X^{4} Z^{2}}=\frac{1}{2}(2-\gamma) \mu \\
& \frac{\ddot{Z}}{Z}+\frac{\dot{X} \dot{Z}}{X Z}+\frac{\dot{Y} \dot{Z}}{Y Z}-a\left(\frac{X}{Y Z}\right)^{2}=\frac{1}{2}(2-\gamma) \mu+\gamma \mu \sinh ^{2} \psi
\end{aligned}
$$

$\left(\gamma \mu X Y Z^{2} \sinh \psi \cosh \psi\right)^{\cdot}=0$

$$
\frac{\dot{X} \dot{Y}}{X Y}+\frac{\dot{Y} \dot{Z}}{Y Z}+\frac{\dot{X} \dot{Z}}{X Z}-\frac{1}{2} a\left(\frac{X}{Y Z}\right)^{2}-\frac{1}{2} \frac{b}{X^{4} Z^{2}}=\mu \cosh ^{2} \psi+(\gamma-1) \mu \sinh ^{2} \psi
$$

and the conservation equations both integrate to give

$$
\begin{aligned}
& \mu^{\gamma} X Y Z \cosh \psi=d \\
& \mu^{(\gamma-1) / \gamma} Z \sinh \psi=e
\end{aligned}
$$

$d, e \in \mathbf{R}$.

We obtain a reduction of these equations for the case $\gamma=2$. By (3.16a) and (3.16b),

$$
\left[Z(X Y)^{\cdot}\right]^{\cdot}=0
$$

which suggests introducing a new time parameter $\xi$

$$
\xi(t)=\int Z^{-1}(t) d t
$$

so that (3.17) may be integrated twice to give

$$
X Y=g\left(\xi-\xi_{0}\right)
$$

$g, \xi_{0} \in \mathbf{R}$.

Using (3.16a), (3.16b) and (3.19), we can now derive an equation for $\mathrm{X}$, which may be written as

$$
W W^{\prime \prime}-W^{\prime 2}+\left(\xi-\xi_{0}\right)^{-1} W W^{\prime}+g\left(\xi-\xi_{0}\right)^{-1} W\left(a W^{3}-b\right)=0
$$

where $^{\prime} \equiv \frac{d}{d \xi}$ and $W=X^{4}\left[g\left(\xi-\xi_{0}\right)\right]^{-1}$.

Equation (3.20) is a special case of the defining equation 


$$
S S^{\prime \prime}-S^{2}-\xi^{-1} S S^{\prime}-\xi^{-1}\left(a_{1} S^{3}+a_{2} S\right)+a_{3} S^{4}+a_{4}=0
$$

of the 3rd Painleve transcendent [6].

Having solved (3.20), we obtain $Y$ from (3.19), and $\psi$ from (3.16f) and (3.16g)

$$
\psi=\operatorname{arctanh}\left[(e g / 2 d)\left(\xi-\xi_{0}\right)\right] .
$$

Now (3.16e) constitutes a linear, first order equation for $Z^{2}$, which may readily be solved to complete the solution.

\section{Extensions}

The method developed in this paper has enabled us not only to obtain in a unified manner the known perfect fluid, Locally Rotationally Symmetric exact solutions, but also to obtain some new solutions. In addition it should be possible to regain the known exact solutions for those L.R.S. spatially homogeneous spacetimes which admit a non-vanishing magnetic field by use of this method (see [4] for references to these solutions).

A natural extension of the results of this paper would be to obtain the decomposability conditions for the case where there are $n$ dependent variables. For $n=3$, this could be applied to the non-L.R.S. spatially homogeneous spacetimes containing a perfect fluid or magnetic field. In this way, it should be possible to regain the known solutions [4], and perhaps to obtain new solutions (one new solution for $n=3$ was given in $\S 3$, where a new choice of dependent variable reduced the problem to $n=2$ ).

It may be that the differential Equation (1.2) occurs in contexts other than the cosmological. In general, the concept of decomposability (corresponding to a restriction on the functional dependence of the integrating factor) could be applied to other types of differential equation (corresponding to operators other than $L_{\mathbf{a}}$ ), to obtain useful integrability conditions.

Acknowledgements. We would like to thank Prof. G. F. R. Ellis for helpful discussions. S. D. N. acknowledges the support of an AECI Research Fellowship, R. M. of U. C. T. and C. S. I. R. scholarships.

\section{References}

1. Ellis, G. F. R., MacCallum, M. A. H. : A class of homogeneous cosmological models. Commun. math. Phys. 12, 108-141 (1969)

2. King, A. R., Ellis, G. F. R. : Tilted homogeneous cosmological models. Commun. math. Phys. 31, 209-242 (1973)

3. Synge, J. L. : Relativity : The general theory, chap. 7. Amsterdam: North-Holland 1960

4. MacCallum, M. A. H. : Cosmological models from a geometric point of view. In: Cargèse lectures in physics, Vol. 6 (ed. E. Schatzman). New York-London-Paris : Gordon and Breach 1973

5. Farnsworth, D. L. : Some new general relativistic dust metrics possessing isometries. J. Math. Phys. 8, 2315-7 (1967)

6. Ince, E. L. : Ordinary differential equations. New York: Dover Publications 1956

Communicated by R. Geroch

Received October 13, 1977 
